\title{
The role of neuroimaging in the ageing brain
}

\begin{abstract}
It is of crucial importance to recognize the changes that occur in the brain ageing. Although neuroimaging studies are important tools used in the evaluation of changes in brain ageing mainly through the exclusion of comorbidities and neurodegenerative disorders, their role is still controversial. In this minireview, we discuss the main aspects of brain ageing, the differences between the young and old brain, the neuroimaging techniques and the use of neuroimaging to assess brain age. Differentiating the differences between normal structural and pathological ageing will provide a deeper understanding of the ageing process of the brain and will bring us new perspectives on biomarkers.
\end{abstract}

Keywords: ageing, neuroimaging, normal brain, brain degenerative disease
Volume 7 Issue 5 - 2017

\section{Patricia Bozzetto Ambrosi,' Marcos Antonio Barbosa da Silva, ${ }^{2}$ Caroline Bozzetto Ambrosi ${ }^{3}$ \\ 'Neuroscientist, Neuroradiologist and Neurosurgeon, Consultant Radiologist and Visiting Professor, France ${ }^{2}$ Radiologist and Neurointervencionist, Hospital Esperan, Brazil ${ }^{3}$ Resident in Internal Medicine at Hospital Moinhos de Vento, Brazil}

Correspondence: Patricia Bozzetto Ambrosi, Neuroscientist, Neuroradiologist and Neurosurgeon, Consultant Radiologist and Visiting Professor, France,

Email patricia.ambrosi@outlook.com

Received: August 29, 2017 | Published: October 10, 2017

\section{Brain aging}

During the lifespan, a number of different structural, functional and molecular changes can happen from innumerable multifactorial changes resulting in the normal neuronal ageing and often in neurodegeneration appearing constantly in the nervous system..$^{1,2}$ It is believed that the ageing process is related to changes in calcium homeostasis that explain the progression of brain ageing. In addition to changes in calcium haemostasis, mitochondrial dysfunction is closely related to neuronal death. ${ }^{3,4}$ Mitochondria are of paramount importance in the generation of adenosine-5-triphosphate, in the sequestration of excess calcium and oxidative stress and the failure of these mechanisms can cause neuronal death. ${ }^{5,6}$ Genetic factors control the susceptibility to calcium and oxidative stress dysregulation and failure of these mechanisms can cause neuronal death.

\section{Differences between the young and old brain}

The Figure 1 shows a comparison between two brain in different age groups. The Figure 1A illustrates a normal appearance and volume of the cortical grooves of a 2 years old child and the Figure 2B shows accentuation of the cortical grooves of the fronto-parietaltemporal regions and cerebellar folds suggesting signs of mild to moderate cerebral aging then it represents the normal aging process of the brain. Therefore, the role of neuroimaging is still considered controversial. ${ }^{8-10}$

Longitudinal studies show all the neurons necessary for the functioning of the organism are already present at birth. The physical development causes the growth of the neurons that increase in size, developing the number and amount of axons and dendrites. ${ }^{11-13}$ Some of these studies estimate the brain mass reaches $75 \%$ of its adult size at 2 years of age. ${ }^{14,15}$ Figure 1 below illustrates the computed sectional images of the brain of a 2-year-old child, showing normal appearance and volume of the cortical grooves and lateral ventricular system.

At the same time, populational studies show that after the age of 40 the volume and weight of the encephalic mass declines considerably, at an estimated rate of $5 \%$ per decade..$^{15}$ It also showed that there is an accentuation in the decline from the seventh decade of life when brain volume decreases by $7 \mathrm{~cm} 3$ each year, with greater loss in frontal and temporal lobes and greater loss of white matter than grey matter in cognitively normal elderly. ${ }^{11-13}$ Cerebral blood flow also decreases heterogeneously by 5 to 20 percent, due to atherosclerosis with the deterioration of mechanisms that maintain cerebral blood flow with the fluctuation of blood pressure. ${ }^{16}$

The ageing-related neuronal loss is most prominent in neurons of the cerebellum and cerebral cortex. While neurons related to the hypothalamic system, brain stem and medullary system present modest losses of neuron or volume. ${ }^{17,18}$ Molecular studies suggest that ageing is a process related to apoptosis (ie, programmed cell death) rather than inflammation, ischemia, or another mechanism ${ }^{2}$. In some areas, however, dendritic connections may increase, perhaps as a result of compensatory plasticity. Although neurons continue to grow new synapses, and new neurons are formed throughout life, but the rates of losses are greater than the gains. Studies have shown the potential protective and restorative effect of exercise may affect brain activity although physiology is still unknown. ${ }^{19,20}$

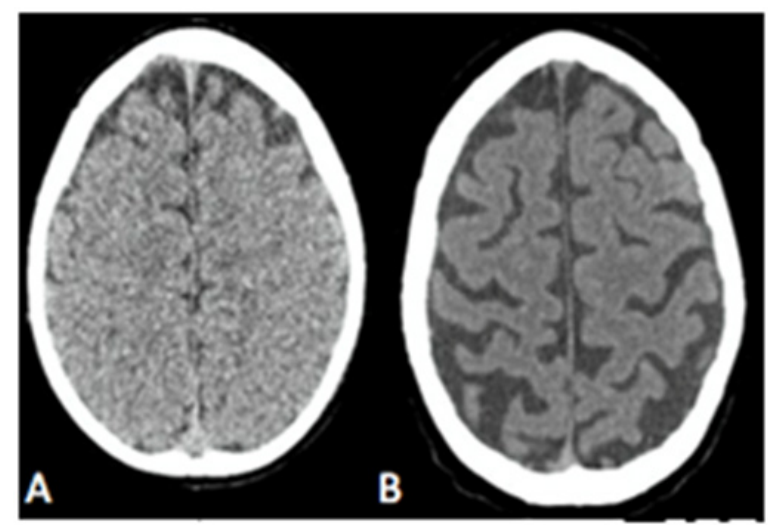

Figure I A In vivo sectional tomographic images illustrating normal development cerebral. B CT axial slice obtainted from 75-year-old adult showing enlargement of the cortical grooves and sylvatic fissures, as well as widening of the grooves between the cerebellar folds and cerebellar fissures, with mild ectasia of the lateral ventricular system, of the 4th ventricle and basal cisterns, due to reduction in brain volume proportional to the age period.

On the other hand, progressive neurodegenerative disorders among which we can highlight Alzheimer's disease, Parkinson's disease, Pick's disease, vascular dementia, Creutzfeldt-Jakob disease 
or Huntington's disease can occur and cause gradual and irreversible deterioration of the cognitive functions with dementia often without apparent detectable cause. ${ }^{21}$

\section{Neuroimaging techniques}

Imaging examinations are one of the main research tools used in practice in the evaluation of neurological problems and cognitive disorders related to brain ageing. Routinely, the neuroimaging studies performed are Computed Tomography (CT) and Magnetic Resonance Imaging (MRI), or also known as structural studies that not only highlight the neurodegenerative aspects but also help exclude other comorbidities that may exacerbate the symptoms.

Despite its low sensitivity and specificity, tending to be more informative than conclusive, the difference between normal and pathological ageing still remains the great challenge. ${ }^{22}$ Generally, neuroimaging research is done as a complementary part of a comprehensive clinical assessment that includes the review of history and medications, physical examination (including neurological and psychiatric components) and assessment of cognitive, functional and behavioural status, blood and urine tests, analysis Cerebrospinal fluid, electroencephalography and other procedures..$^{9,10}$

Other imaging modalities also important are functional imaging studies including Magnetic Resonance Spectroscopy (MRS) and other functional modalities and molecular studies that we will discuss later in this chapter. MRS uses magnetic resonance imaging techniques to measure metabolic activity in the region of interest. MRS has a lower resolution than structural magnetic resonance imaging, but it allows monitoring aspects of the intermediate metabolism that are not obtained by other methods in vivo. ${ }^{9}$

\section{Use of neuroimaging to assess the age of the brain}

The most relevant structural radiological findings identified from CT and/or MRI in cerebral ageing are, among others:

\section{i. Cerebral/hippocampal atrophy, \\ ii. Ventriculomegaly, \\ iii. Cerebrovascular disorders \\ iv. Presence of microbleeds.}

As these findings are identified in both normal and pathological ageing, although typically age-related changes are characterized by being less rapid and generally less severe than those of neurodegenerative diseases. ${ }^{8}$

Cerebral atrophy is manifested by enlargement of the grooves, narrowing of the gyri, thinning of the grey matter, decreased white matter volume and/or enlargement of the cerebral ventricles and subarachnoid spaces. Asymmetric cortical atrophy is often observed in neurodegenerative dementias and sometimes it manifests as disproportionate deficiencies in functions mediated by the most atrophic regions. Hippocampal atrophy is one of the earliest and most prominent manifestations of $\mathrm{AD}$ although it is not pathognomic of $\mathrm{AD}$, it may occur in other conditions such as mesial temporal sclerosis, temporal lobe epilepsy and certain vascular insults. Certain regions of the cerebral parenchyma show selective vulnerability to different neurodegenerative disorders. ${ }^{22}$ MRI imaging has more specificity than CT and automated determination of hippocampal volume in MRI is increasingly available and may be useful in determining the probability of pathological atrophic change.
Ventricular enlargement may occur in association with progressive cortical atrophy without evidence of obstruction; This is known as ex-vacuum hydrocephalus. ${ }^{89}$ Alternatively, ventricular enlargement may occur in association with normal pressure hydrocephalus (NPH). Recent advances in imaging studies have made it possible to distinguish these two pathological entities. NPH is a potentially treatable cause of dementia that usually presents as the symptomatic triad including dementia, incontinence, and gait disturbance. ${ }^{23}$

Cerebrovascular disease associated with brain ageing may manifest itself through cognitive deficits ranging from mild impairment to frank dementia. ${ }^{24}$ The increasing prevalence of ischemic white matter changes with advancing age has been implicated as one of the factors responsible for the age-related cognitive decline. The most common types of vascular cognitive impairment manifest visible changes in structural imaging studies of the brain. Other common findings associated with cognitive impairment are related to cerebrovascular disease include cortical and subcortical infarcts and periventricular white matter lesions. In Figure 2, we observed an MRI study illustrating subtle alterations in the periventricular white matter related to normal ageing. Serial neuroimaging studies can sometimes be used to document the temporal relationship between cerebrovascular changes and the onset or progression of cognitive impairment.

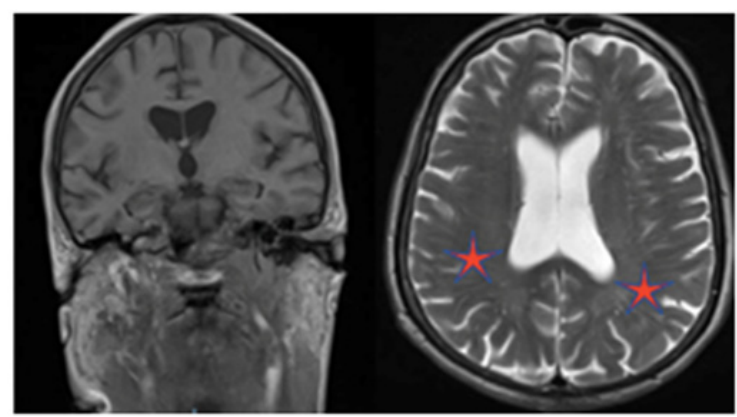

Figure 2 In vivo sectional MR images of the brain illustrating bilateral mild degree microangiopathy.

Cerebral microbleeds that may occur in association with vascular anomalies, amyloid angiopathy, and hypertensive microangiopathy are a common MRI finding in older individuals, particularly visualized from special gradient echo and susceptibility imaging techniques. ${ }^{24}$ In population studies, brain microbleeds are detected in approximately 15 to 25 percent of individuals over 75 years of age and become more common with advancing age. ${ }^{25,26}$ When associated with amyloid angiopathy are primarily visualized in the cerebral cortex and are usually associated with more severe cognitive impairment. On the other hand, microbleeds associated with small vessel disease typically occur in the basal ganglia, thalamus or bridge. Both are associated with increased risk of bleeding and should be investigated in patients with indication of anticoagulant use.

Functional neuroimaging includes functional imaging and nuclear medicine studies such as Positron Emission Tomography (PET) and Single Photon Emission Computed Tomography (SPECT). The molecular neuroimaging derives from Nuclear Medicine where they are molecular images coupled to SPECT and PET. Both techniques are used in patients with suspected dementia or already established. They measure parameters that are coupled or correlated with the neuronal activity of the brain. These techniques are only available only in specialized centres and many are only used at the research level. In clinical practice, most functional and molecular images are interpreted by visual inspection, although the parameters to be measured are inherently quantifiable. Recognition of regional patterns 
of hypoperfusion or hypometabolism in functional images may aid in the differential diagnosis of various forms of dementia.

Examples of molecular neuroimaging modalities include 123I-FPCIT Single Photon Emission Tomography (DAT) scans used for confirmation of Parkinson's disease and amyloid PET imaging in AD. Image of the striatal dopamine transporter - DAT exams use the ioflupane iodine-123 compound to measure the presynaptic dopamine transport in the brain. ${ }^{27}$ New molecular techniques are now under development to detect other pathological processes associated with dementia, such as neuroinflammation and deposition of tau protein. ${ }^{28}$ Other functional imaging studies include measures of cerebral perfusion or blood flow and cerebral metabolic activity.

Brain ageing involves a number of multifactorial events that occur over the years as a result of molecular and cellular factors that result in processes of neuronal degeneration. Advances in neuroimaging techniques have allowed us to broaden the field of research and new treatments for several pathologies including those arising and/or related to the processes of normal or pathological ageing. Although our challenge is still to differentiate between normal structural and pathological ageing, functional models should help us find biomarkers that will make us better understand this process.

\section{Acknowledgements}

None.

\section{Conflict of interest}

None.

\section{Funding}

None.

\section{References}

1. Mrak RE, Griffin ST, Graham DI. Aging-associated changes in human brain. J Neuropathol Exp Neurol. 1997;56(12):1269-1275.

2. Lourenço CF, Ledo A, Barbosa RM, et al. Neurovascular- neuroenergetic coupling axis in the brain: master regulation by nitric oxide an consequences in aging and neurodegeneration. Free Radic Biol Med. 2017; 108:668-682.

3. Brini $\mathrm{M}, \mathrm{Calì} \mathrm{T}$, Ottolini $\mathrm{D}$, et al. Neuronal calcium signaling: function and dysfunction. Cell Mol Life Sci. 2014;71(15):2787-2814.

4. Nicholls DG. Oxidative stress and energy crises in neuronal dysfunction. In: GE Gibson et al. (Eds.), New York Academy of Sciences, WileyBlackwell, New York, NY, USA. 2008. p.53-60.

5. Foster TC, Kumar A. Calcium dysregulation in the aging brain Neuroscientist. 2002;8(4):297-299.

6. Adams HH, Hibar DP, Chouraki V, et al. Novel genetic loci underlying human intracranial volume identified through genome-wide association. Nat Neurosci. 2016;19(12):1569-1582.

7. Rastogi S, Borgo B, Pazdernik N, et al. Caenorhabditis elegans glp4 Encodes a Valyl Aminoacyl tRNA Synthetase. G3 (Bethesda). 2015;5(12):2719-2128.

8. Murray AD. Imaging in Alzheimer's disease and other dementias. Preface Neuroimaging clinics of North America 22(1): xiii-xiv. 2012.

9. Fritz JV. Neuroimaging trends and future outlook. Neurologic clinics. 2014;32(1):1-29.
10. Bertelson JA, Ajtai B. Neuroimaging of dementia. Neurol clin. 2014;32(1):59-93.

11. Mueller EA, Moore MM, Kerr DC, et al. Brain volume preserved in healthy elderly through the eleventh decade. Neurology. 1998;51(6):1555-1562.

12. Salat DH, Kaye JA, Janowsky JS. Prefrontal gray and white matter volumes in healthy aging and Alzheimer disease. Arch Neurol. 1999;56(3):338-344.

13. Tang Y, Whitman GT, Lopez I, et al. Brain volume changes on longitudinal magnetic resonance imaging in normal older people. $J$ Neuroimaging. 2001;11(4):393-400.

14. Spear LP. The role of brain development in drug effect and drug response. In: PM Miller, SA Ball, ME Bates, et al, (Eds.), Elsevier Academic Press, San Diego, CA, US. 2013. p.271-280.

15. Driscoll I, Davatzikos C, An Y, et al. Longitudinal pattern of regional brain volume change differentiates normal aging from MCI. Neurology. 2009;72(22):1906-1913.

16. Wagner M, Jurcoane A, Volz S, et al. Age-related changes of cerebral autoregulation: new insights with quantitative T2'-mapping and pulsed arterial spin-labeling MR imaging. AJNR Am J Neuroradiol. 2012;33(11):2081-2087.

17. Leyton M, Kennedy SH. Neuroimaging tests for clinical psychiatry: Are we there yet? J Psychiatry Neurosci. 2017;42(4):219-221.

18. Baloch S, Verma R, Huang H, et al. Quantification of brain maturation and growth patterns in c57BL/6J mice via computational neuroanatomy of diffusion tensor images. Cereb Cortex. 2009; 19(3):675-687.

19. Buchman AS, Boyle PA, Yu L, et al. Total daily physical activity and the risk of $\mathrm{AD}$ and cognitive decline in older adults. Neurology. 2012;78(17):1323-1329.

20. Oliveira BF, Nogueira-Machado JA, Chaves MM. The role of oxidative stress in the aging process. Scientific World Journal. 2010;10:1121-1128.

21. Thiel GM, Ambrosi PB, Dall'Agnoll O. Alzheimer, a doenÁa do sĖculo: dos sintomas aos fatores de risco^ipt". Rev cient AMECS. 1999;8(1):20-23.

22. Konrad C, Ukas T, Nebel C, et al. Defining the human hippocampus in cerebral magnetic resonance images-An overview of current segmentation protocols. Neuroimage. 2009;47(4):1185-1195.

23. Algin O, Hakyemez B, Taskapilioglu O, et al. Morphologic features and flow void phenomenon in normal pressure hydrocephalus and other dementias: are they really significant Acad Radiol . 2009;16(11):1373-1380.

24. Charidimou A, Boulouis G, Gurol ME, et al. Emerging concepts in sporadic cerebral amyloid angiopathy. Brain. 2017;140(7):1829-1850.

25. Boulouis G, Charidimou A, Greenberg SM. Sporadic Cerebral Amyloid Angiopathy: Pathophysiology, Neuroimaging Features, and Clinical Implications. Semin Neurol. 2016;36(3):233-243.

26. Vernooij MW, Smits M. Structural neuroimaging in aging and Alzheimer's disease. Neuroimaging Clin N Am. 2012;22(1):33-55.

27. Brigo F, Turri G, Tinazzi M. 123I-FP-CIT SPECT in the differential diagnosis between dementia with Lewy bodies and other dementias. $J$ Neurol Sci. 2015;359(1-2):161-171.

28. Niedernhofer LJ, Kirkland JL, Ladiges W. Molecular pathology endpoints useful for aging studies. Ageing Res Rev. 2017;35:241-249. 